\title{
GESELLSCHAFTLICHE VERANTWORTUNG ALS GRUNDLAGE EINES NACHHALTIGEN UNTERNEHMENS
}

\begin{abstract}
Die Konkurrenzfähigkeit im Wirtschaftshandeln wird heute bei vielen Wirtschaftsorganisationen nicht alleine auf der Grundlage der Realisierung entsprechender ökonomischer Werte oder durch Erreichung bestimmter „innerer” Werte wie Qualitätsstandards bei Waren oder Dienstleistungen u. ä. aufgebaut. Für eine erfolgreiche Unternehmensführung ist vielmehr die Erfüllung bestimmter ,äußerer” Anforderungen ausschlaggebend. Eine dieser ,äußeren” Anforderungen, die an unterschiedliche Formen des unternehmerischen Handelns gestellt werden, ist heute die Notwendigkeit einer nachhaltigen Entwicklung. Die Erfüllung der Anforderungen einer nachhaltigen Entwicklung erfordert eine weitgehende Verbindung der ökonomischen Ziele und Werte mit den ökologischen und gesellschaftlichen Anliegen. Eine der wesentlichen Grundlagen hierfür, die zugleich ein Mittel zur Gestaltung eines nachhaltigen Unternehmentums kann das Konzept einer gesellschaftlichen Verantwortung der Unternehmen (Corporate Social Responsibility, CSR) abgeben, das mittlerweile mit komplexen Indikatorensystemen und entsprechenden Regelwerken (z.B. ISO 26000) arbeitet. Der vorliegende Beitrag behandelt die wichtigsten CSR-Ansätze sowohl als Grundlage für nachhaltige Betriebswirtschaft als auch als möglichen Beitrag der Unternehmen zur nachhaltigen Entwicklung überhaupt. Dabei wird von einer Einsicht ausgegangen, dass das Prinzip gesellschaftlich verantwortlichen Wirtschaftens nicht nur die Wirtschaftsorganisationen selbst, sondern vielmehr auch ihr soziales, politisches und kulturelles Umfeld vor neue Herausforderungen stellt. Im Beitrag werden die wichtigsten Barierren identifiziert, die eine erfolgreiche Umsetzung der Grundsätze nachhaltiger Entwicklung und gesellschaftlicher Verantwortung der Wirtschaft erschweren oder gar verhindern. Dabei wird auf zahlreiche strukturelle Ähnlichkeiten zwischen beiden Bereichen verwiesen. Aus der Idee einer gesellschaftlichen Verantwortung der Wirtschaft entstanden - ähnlich wie früher beim Leitbild nachhaltiger Entwicklung viele Visionen, die eine Reihe von Kontroversen sowohl auf der theoretischen als auch auf der praktischen Ebene erwecken. In den bisherigen Diskussionen ist es nicht gelungen, einen theoretischen Konsens über ein angemessenes Verständnis von Verantwortung in Bezug auf kollektive und korporative (institutionelle) Akteure zu erzielen. Der wichtigste Grund dafür ist die verbreitete traditionelle Auffassung, dass nur individuelle Subjekte verantwortungsfähig sind. Die Folgen sind ernsthafte Probleme, die bei der Umsetzung der Idee einer gesellschaftlichen Verantwortung der Unternehmen auf der Ebene der Alltagspraxis entstehen. Zwar werden durch die Verknüpfung der Idee der gesellschaftlichen Verantwortung von Unternehmen mit Nachhaltigkeitsgedanken nicht alle diese Probleme gelöst, doch durch eine paralelle Verwirklichung beider Leitbilder können jedoch viele positiven Synergien und „Mehrwerte“ erzeugt werden, die bei einer Separierung beider Bereiche gar nicht denkbar sind.
\end{abstract}

\footnotetext{
${ }^{1}$ Prof. dr hab. Andrzej Kiepas, Zakład Antropologii Filozoficznej i Filozofii Cywilizacji, Uniwersyetet Śląski, ul. Bankowa 11, 40-007 Katowice, e-mail: andrzej.kiepas@us.edu.pl
} 
Schlüsselbegriffe: Nachhaltigkeit, Öko-Entwicklung, gesellschaftliche Verantwortung von Unternehmen, Wirtschaftsethik

Die Versuche der Wirtschaftskontrolle hingen immer mit bestimmten Problemen und Kontroversen zusammen, die u.a. die Notwendigkeit ihrer Anwendung und die Verwirklichungsweisen betrafen. Die durch die Wirtschaftsentwicklung verursachten Folgen, die schließlich einen zivilisationellen Charakter haben, führten zur Situation, dass die Fragen nach der Notwendigkeit der Kontrolle nicht bestritten wurden. Das Bewusstsein der globalen Bedrohungen wurde auch mit der Akzeptanz der notwendigen globalen und lokalen Veränderungen verbunden. Man soll damit die Prozesse der Zivilisationsentwicklung und ihre Folgen auf bestimmte Weise kontrollieren, und die Nachhaltigkeit bleibt in dieser Hinsicht eine Idee, die Regulationscharakter haben kann. Grundlegende Bedeutung für nachhaltige Entwicklung hat das Prinzip der intra- und intergenerationellen Gerechtigkeit. Es verbindet sich damit das Hauptziel der nachhaltigen Entwicklung, nämlich die Erhaltung der Möglichkeit der weiteren Entwicklung nicht nur in kurzer Perspektive, sondern auch für die zukünftigen Generationen. Die Idee der Nachhaltigkeit hat auch die Diskussion über ihre Kriterien, Indikatoren und Verwirklichungsweisen hervorgebracht. Sie verbindet sich nämlich mit vielen spezifischen Zielen, und man braucht immer die Mittel, um die Verwirklichung solcher Ziele zu bestimmen und einzuschätzen, was auch das Allgemeinziel der Nachhaltigkeit betrifft $^{2}$. Die Idee der Nachhaltigkeit wird heute sehr breit und in Verbindung mit unterschiedlichen Aspekten durch Vertreter verschiedener Wissenschaften diskutiert. Es entwickelt sich auch die Diskussion, die die Deutung und das Verständnis von Nachhaltigkeit betrifft. Die Idee der Nachhaltigkeit, die im allgemeinen ziemlich einfach ist, verbindet sich jedoch mit vielen einzelnen und spezifischen Problemen, die man unterschiedlich sehen und einschätzen kann. Nachhaltigkeit bestimmt das allgemeine Ziel, das mit der Möglichkeit der weiteren Enwicklung zusammenhängt. Diese Idee entscheidet jedoch nicht, auf welche Weise dieses Ziel erreichbar sein soll und welche Faktoren entscheidende Bedeutung für die Verwirklichung bzw. für die Behinderung dieses Zieles haben. Es wird damit auch nicht über qualitative Kriterien der Entwicklung entschieden. Die Idee der Nachhaltigkeit weist auf ein allgemeines Ziel hin, aber es stellt das auf eine wertneutrale Weise vor, und das verursacht, dass die qualitativen Inhalte dieser Idee nur auf eine bestimmte, nicht auf eine beliebige Weise erfüllt werden soll. Es gibt viele Faktoren, die in dieser Hinsicht eine wichtige Rolle spielen. Man kann eventuell auch die wichtigsten Faktoren vorstellen, aber diese Wahl wird immer mit Willkür verbunden sein, was auch zu den bestimmten negativen Folgen führen kann. Die bisherige Diskusssion, die in dieser Hinsicht in Polen stattfand, wurde durch die ökologischen Aspekte dominiert. Sie wurde mit der Idee der Ökoentwicklung verbunden, obwohl die ökologische Faktoren zwar wichtig, aber nicht entscheidend sind.

Die Idee der Nachhaltigkeit verbindet sich mit den bestimmten Erforderungen, vor allem mit folgenden:

\footnotetext{
${ }^{2}$ Vgl.: T. Borys (Hg.): Wskaźniki ekorozwoju (Indikatoren der Ökoentwicklung). Białystok 1999; Idem (Hg.): Wskaźniki zrównoważonego rozwoju (Indikatoren nachhaltiger Entwicklung). Warszawa-Białystok. 2005. Vgl. auch: A.Papuziński: Nachhaltigkeitsdeutungen in der polnischen wissenschaftlichen Fachliteratur, in: G. Banse, A. Kiepas (Hg.): Nachhaltige Entwicklung. Von der wissenschaftlichen Forschung zur politischen Umsetzung. Berlin 2005, S. 105ff.
} 
1. Notwendigkeit der ganzheitlichen Einstellung - die Entwicklung hat einen ganzheitlichen Charakter, was die Notwendigkeit eines holistischen Blicks aufdrängt;

2. Konkretheit - in einer Situation, in der es viele wichtige Faktoren gibt, ist ihre Anerkennung und Verknüpfung von entscheidender Bedeutung;

3. Erhaltung der Differenzheit - man kann Nachhaltigkeit nicht auf bestimmte Aspekte oder Faktoren reduzieren; in diesem Fall bedeutet Differenzheit die gleichzeitige Bedeutsamkeit vieler Faktoren.

Die Erfüllung dieser Anforderungen ist weder eindeutig noch leicht und ist zugleich mit vielen Konflikten verbunden. Die Notwendigkeit der ganzheitlichen Einstellung muss sich mit der Auswahl der wesentlichen Aspekte verbinden, die für diese Ganzheit entscheidend sind. Das kann sich auch mit solchen Auswahlen verbinden, die etwas Wichtiges aus dem Blickfeld verlieren oder nicht berücksichtigt können, oder etwas, was z.B. heute noch keine so große Rolle spielt, aber in der Zukunft von wesentlicher Bedeutung sein kann. Z. Piatek weist in dieser Hinsicht auf drei Sachgebiete hin, die für die Verwirklichung von Nachhaltigkeit entscheidende Bedeutung haben: (a) die Ökoentwicklung als das Sachgebiet der Beziehungen zwischen Mensch und Natur, (b) das Sachgebiet der Wirtschaft und (c) das politisch-gesellschaftliche Sachgebiet ${ }^{3}$. Man weist gleichzeitig auf bestimmte Grundlagen hin, die mit der Idee der Nachchaltigkeit zusammenhängen, vor allem auf folgende:

- $\quad$ „die Voraussetzung über den offenen Charakter der geschichtlichen Prozesse, die die notwendige Bedingung für die Möglichkeit der Gestaltung von Geschichte durch menschliche Gattung, homo sapiens, betrifft;

die Voraussetzung über die Koevolution zwischen der biologischen und gesellschaftlich-kulturellen Natur vom Menschen, was die Bedingung der Modifikation und vielleicht auch der Vervollkommnung von biologisch bedingten menschlcihen Neigungen bedeutet;

- $\quad$ wesentlich wichtig ist auch die Bemerkung, dass die Bildung der Nachchaltigkeit im Bereich der Anthroposphäre, die in Richtung der Symbiose mit der Natur geht, auf verschiedene Weise verwirklicht sein kann, und deshalb die Nachhaltigkeit zeichnet nicht direkt konkrete Arten der Zivilisation aus [...]"4.

Die Idee der nachhaltigen Entwicklung erscheint heute im Kontext unterschiedlicher Veränderungen und deshalb soll das Verständnis von Nachhaltigkeit auch diese Kontexte berücksichtigen. Man kann dazu folgende rechnen:

1. Die kulturellen und gesellschaftlichen Veränderungen, die mit dem Durchbrechen der modernen und mit der Gestaltung der postmodernen Ordnung zusammenhängen - damit verbundene Veränderungen hängen u.a. mit der gesellschaftlichen und kulturellen Rolle von Wissenschaft und Technik zusammen.

2. Die Prozesse der Globalisierung und die damit verbundenen Folgen - wichtig ist ihr globales Ausmaß, das gleichzeitig mit vielen spezifischen Ereignissen verbunden ist.

3. Die Vergrösserung der Rolle von Risiko und Ungewissheit in der Perspektive der sog. „Risikogesellschaft“ - eine der in dieser Hinsicht wichtigen Konsequenzen ist die Notwendigkeit der Akzeptanz eines bestimmten Niveaus und der gerechtigen Verteilung des Risiko.

\footnotetext{
${ }^{3}$ Vgl. Z. Piątek: Ekofilozofia (Ökophilosophie). Kraków 2008, S. $151 \mathrm{ff}$.

${ }^{4}$ Ibidem, s. 156.
} 
4. Die Entwicklung der Informationstechnologien und die Perspektive der Informationsgesellschaft - diese Problematik ist bis heute im Kontext der Verwirklichung der Prinzipien der Nachhaltigkeit am wenigsten erforscht.

Die Verwirklichung der Idee der Nachhaltigkeit verbindet sich im Allgemeinen mit der entsprechenden Gestaltung von drei grundsätzlichen Ordnungen: einer gesellschaftlichen, einer wirtschaftlichen und einer ökologischen Ordnung ${ }^{5}$. Die Verknüpfung dieser drei Bereiche erfordert die Überwindung unterschiedlicher partikularer Handlungen und Sichtweisen. Die für die konkreten Sachgebiete bestimmten und auf die konkreten Sachgebiete begrenzten Handlungen sind ungenügend in bezug auf ganzheitliche Erfordernisse, die mit Nachhaltigkeit verbunden werden. Die Gestaltung der wirtschaftlichen Ordnung hat keine große Bedeutung im Verhältnis $\mathrm{zu}$ den anderen Ordnungen, aber in bezug auf die Bedeutung wirtschaftlicher Faktoren und ihrer Ordnung erreicht sie eine besondere Gültigkeit. Die oben genannten Kontexte spielen auch eine gewisse Rolle in der wirtschaftlichen Ordnung, wo sie konkrete Inhalte erreichen und sich mit den notwendigen Veränderungen, die die wirtschaftliche Ordnung betreffen, verbinden. Nachhaltigkeit erfordert in dieser Hinsicht auch Veränderungen in Bezug auf unterschiedliche Ebenen der wirtschaftlichen Ordnung: von der globalen über internationale und nationale Ordnungen bis hin $\mathrm{zu}$ den lokalen wirtschaftlichen Institutionen (Unternehmen, Banken usw.). Im Fall der wirtschaftlichen Organisationen handelt es sich um die Notwendigkeit der Herausbildung einer nachhaltigen, d.h. mit den Erforderungen der Nachhaltigkeit übereinstimmenden Unternehmenskultur.

Die anderen Aspekte heutiger Veränderungen und der mit der Wirtschaft verbundenen Erfordernisse hängen auch mit der Notwendigkeit der Verknüpfung der Wirtschaft mit der Ethik sowie mit der Institutionalisierung der Ethik in der Wirtschaft zusammen. Die in dieser Hinsicht wichtigste Idee ist die Konzeption der gesellschaftlichen Verantwortung (corporate social responsibility), deren Verwirklichung in der Wirtschaft mit vielen Herausforderungen und Problemen zusammenhängt. Die Konzeption der korporativen gesellschaftlichen Verantwortung verbindet sich hier mit der Idee der Nachhaltigkeit, was zusätzlich zu vielen theoretischen und praktischen Problemen führt. Die Verwirklichung dieser beiden Konzeptionen führt schließlich zur notwendigen Veränderung im Bereich der wirtschaftlichen Ordnung. Die Konzeption der korporativen gesellschaftlichen Verantwortung ist u.a. mit folgenden Problemen verbunden:

a) theoretische Probleme- die Beziehungen zwischen Wirtschaft und Ethik; die Rolle der Ethik als ein Regulierungsfaktor für die Wirtschaft; das Verständnis der korporativen gesellschaftlichen Verantwortung und ihrer Erfordernissen;

b) praktische Probleme- diese hängen mit der Notwendigkeit der Verwirklichung und Institutionalisierung der Prinzipien von der korporativen gesellschaftlichen Verantwortung in der Praxis der bestimmten wirtschaftlichen Organisationen.

Analoge Probleme sind auch im Fall der Verwirklichung von Konzeptionen der Nachhaltigkeit zu bemerken. Es gibt schließlich auch das allgemeine Problem, das mit den Beziehungen zwischen Nachhaltigkeit und korporativer gesellschaftlicher Verantwortung verbunden ist. Es scheint, dass diese Beziehungen selbstverständlich sind und man sie nicht genauer zu analysieren braucht. Die Beziehungen zwischen Ethik und Wirtschaft

\footnotetext{
${ }^{5}$ Vgl. J. Kronenberg,T. Bergier (Hg.): Wyzwania zrównoważonego rozwoju w Polsce (Herausforderungen nachhaltiger Entwicklung in Polen), Kraków 2010, S. $223 \mathrm{ff}$.
} 
sind der Gegenstrand der Wirtschaftsethik, in der auch die Konzeption der korporativen gesellschaftlichen Verantwortung entstand und diskutiert wurde. Diese Konzeption war schon von Anfang an mit unterschiedlichen Streitfragen und Einstellungen verbunden. Die Kontroversen betrafen z.B. auf der theoretischen Ebene die Fragen, ob man kollektiven Subjekten Verantwortung zuschreiben kann. Die Konzeption der korporativen gesellschaftlichen Verantwortung hängt u.a. mit folgenden zwei Aspekten zusammen:

a) Die Erweiterung des Objektbereichs der Unternehmensverantwortung, die bisher durch den Rahmen der Funktionsweisen des Unternehmens begrenzt wurden - in dieser Hinsicht handelt es sich nicht nur um die Erhöhung des Profits und um die Entwicklung bestimmter Bestände des Unternehmens sowie um die Gestaltung der interpersonalen Relationen, sondern sie soll als Objekt der Verantwortung auch die äußere, naturale und gesellschaftliche, Umgebung umfassen;

b) das Subjekt der Verantwortung - Verantwortung betrifft nicht nur individuelle Subjekte, sonden auch die Organisation (Institution) als Ganzes; es handelt sich in dieser Hinsicht nicht um die rechtliche, sondern um eine moralische Verantwortung.

Theoretische Probleme der korporativen gesellschaftlichen Verantwortung betreffen vor allem die Fragen nach dem Subjekt solcher Verantwortung, weil man traditionell nur individuellen Subjekten Verantwortung zuschreiben kann. Die Institutionalisierung der wirtschaftlichen Tätigkeit führte schließlich zu einer Situation, in der ein Missklang zwischen dem Subjekt der Handlung und dem Subjekt der Verantwortung entsteht. Das Individuum wurde traditionell als Subjekt der Handlung als auch als Subjekt der Verantwortung verstanden, aber heute handeln die Individuen im Rahmen von Institutionen, die gleichzeitig Subjekte der Verantwortung sein sollen. Die Fragen nach der institutionellen Mitverantwortung wurden auch Gegenstand der Diskussionen in der Wirtschaftsethik ${ }^{6}$. Das Problem ist schwierig und nicht leicht zu lösen, und deshalb haben wir bis heute keine richtige Konzeption der korporativen gesellschaftlichen Verantwortung. Die Diskussion in der Wirtschaftsethik hat gezeigt, dass zwar sowohl die Anhänger als auch die Gegner dieser Verantwortung ähnliche Argumente benutzt haben, aber jeweils andere Schlussfolgerungen aus diesen Voraussetzungen abgeleitet wurden. Es sieht im Moment so aus, dass dieser Streit theoretisch nicht lösbar ist, weshalb die Probeleme der korporativen gesellschaftlichen Verantwortung auf die praktische Ebene verlagert wurden. Versuche, die Erfordernisse der korporativen gesellschaftlichen Verantwortung zu erfüllen, sind auch mit der Notwendigkeit der Veränderung des Status von Unternehmen sowie, breiter, mit fundamentalen Veränderungen der Ökonomie selbst verbunden. Darauf verweist z.B. die Konzeption des Unternehmens als eine Organisation, die in Übereinstimmung mit der Erfüllung der Bedürfnisse sog. interessierter Personen (stakeholders) handelt, hin $^{7}$. Die praktische Probleme der Verwirklichung der Konzeption korporativer gesellschaftlicher Verantwortung hängen auch breiter mit den Problemen der Institutionalisierung von Ethik in der Wirtschaft, z.B. mit der Verwirklichung ethischer Stardards, zusammen, die in der internationalen Norm der gesellschaftlichen

\footnotetext{
${ }^{6}$ Bestimmte Einstellungen kann man u.a. finden in: L. V. Ryan, J. Sojka (Hg.): Etyka biznesu. Z klasyki współczesnej myśli amerykańskiej (Wirtschaftsethik.Vom klassischen modernen amerikanischen Denken). Poznań 1997.

${ }^{7}$ Vgl. W. M. Evan, R. E. Freeman: Spółka i osoby żywotnie zainteresowane. Kapitalizm kantowski (Aktiengesellschaft und Stakeholder. Kantischer Kapitalismus), w: L. V. Ryan, J. Sojka (red.): Etyka biznesu (Wirtschaftsethik), op. cit., s.185-205.
} 
Verantwortung ISO 26000 vorgestellt wurden. Diese Norm ist bis heute eine freiwillige Norm, die nicht mit bestimmten Zertifizierungsprozessen verbunden ist und als Ziel lediglich die Sensibilisierung bestimmter Subjekte und ihrer Neigung zur Erfüllung konkreter Prinzipien hat. In dieser Norm wird verwiesen auf

- $\quad$ die Prinzipien und Prozeduren, die die gesellschaftliche Verantwortung betreffen; - $\quad$ die Bereiche und Probleme, die einen Schlüsselcharakter in diesem Bereich haben;

- $\quad$ die Aufgaben im Bereich der Implementierung, Gestaltung und Beförderung der Prinzipien korporativer gesellschaftlicher Verantwortung in unterschiedlichsten Organisationen und auch in der Umgebung, in der sich die Einflüsse der Organisation äussern;

die Notwendigkeit der Vertiefung der Beziehungen mit den, für jede Organisation charakteristischen, interessierten Personen;

- die Verbreitung der Ideen und der guten Handlungsweisen (best practice) im Bereich der gesellschaftlichen Verantwortung ${ }^{8}$.

Schlüsselbereiche, die mit den unterschiedlichen Ebenen der organisatorischen Tätigkeit zusammenhängen, sind:

- die Menschenrechte;

- $\quad$ das Verwalten von Humanressourcen;

- $\quad$ die Umweltprobleme;

- $\quad$ ehrliche Wirtschaftspraktiken;

- $\quad$ die Bedürfnise der Verbraucher;

- $\quad$ die Gestaltung und Entwicklung der Gesellschaft.

Handlungen in Übereinstimmung mit den Normen der gesellchaftlichen Verantwortung sollen zusätzlich auch folgende Prinzipien berücksichtigen: Transparenz, ethisches Handeln, Verantwortung, Achtung der Rechtsvorschriften, Achtung der Rechte von Stakeholdern sowie der internationalen Normen und der Menschenrechte ${ }^{9}$. Die Verwirklichung der Konzeption der korporativen gesellschaftlichen Verantwortung erfordert gleichzeitig die Erfüllung vieler konkreter Bedingungen. Die Gestaltung dieser Verantwortung ist mit vielen Schwierigkeiten und Begrenzungen verbunden, obwohl sie gleichzeitig für die Organisation unterschiedliche Vorteile haben kann, etwa:

- Sie erlaubt die Erhaltung eines bestimmten guten Rufes und des Konkurrenzvorteils der Organisation;

- $\quad$ sie erhöht die Attraktivität der Organisation für die Mitarbeiter, aber auch die Produktivität der Organisation und ihre Attraktivität für Kunden und Kontrahenten;

- $\quad$ sie verbessert die Einschätzung der Organisation auf dem Markt und in bestimmten gesellschaftlichen Milieus;

\footnotetext{
${ }^{8} \mathrm{Vgl}$. M. Rybak, Etyka menedżera - społeczna odpowiedzialność przedsiębiorstwa (Managerethik gesellschaftliche Verantwortung vom Unternehmen). Warszawa 2004; W. Gasparski (Hg.): Europejskie standardy etyki i społecznej odpowiedzialności biznesu (Europäische Standards der Ethik und der gesellschaftlichen Verantwortung). Warszawa 2003; W. Ocieczek, B. Gajdzik: Społeczna odpowiedzialność przedsiębiorstw produkcyjnych (Gesellschaftliche Verantwortung von Produktionsunternehmen). Gliwice 2010; D. Fobelova, P. Fobel: Etyka i kultura w organizacji (Ethik und Kultur in der Organisation). Tychy 2007. Vgl. auch: www.pkn.pl/iso-26000; www.iso.org/iso/iso26000.

${ }^{9} \mathrm{Vgl}$. www.pkn.pl/iso-26000; www.iso.org/iso/iso26000.
} 
- $\quad$ sie hat positive Einflüsse auf die Relationen mit den direkten und indirekten Stakeholdern;

- $\quad$ sie kann das Niveau der Innovationskraft erhöhen.

Die Verwirklichung der Konzeption der korporativen gesellschaftlichen Verantwortung kann ein Faktor der Erhöhung der Kultur von Organisationen sein, was sich in der Erhöhung der Effektivität bestimmter Handlungen und Entscheidungen äußert. Es können sich damit auch die moralische Kultur und die Beziehungen mit der Umgebung erhöhen.

Probleme der Innovationsfähigkeit werden heute auf der Grundlage unterschiedlicher Perspektiven diskutiert; man spricht in dieser Hinsicht z.B. über verantwortbare Innovationen (responsible innovations). Man muss bestimmte Innovationen ganzheitlich einschätzen und bei dieser Gelegenheit unterschiedliche Kriterien benutzen: technische, ökonomische, ökologische, gesellschaftliche und kulturelle. Die Konzeption der korporativen gesellschaftlichen Verantwortung kann auch in dieser Hinsicht eine gewisse fördernde Rolle spielen. Sie verbindet sich auch hier mit der Konzeption nachhaltiger Entwicklung, was in der Norm ISO 26000 betont wird ${ }^{10}$. Man kann die Beziehungen zwischen diesen beiden Konzeptionen erkennen, wenn man die Prinzipien der Nachhaltigkeit anschaut, die für wirtschaftliche Organisationen wichtig sind.

Zur Verwaltung solcher Organisationen in Übereinstimmung mit der Konzeption der Nachhaltigkeit gehören vor allem folgende Prinzipien ${ }^{11}$ :

1. „Die Verwirklichung innovativer Lösungen im Bereich der nachhaltigen Entwicklung ist ein Teil des gesellschaftlichen Auftrags der Unternehmen“12 - man stellt in dieser Hinsicht unterschiedliche Modelle vor und im Allgemeinen kann man sagen, dass die Probleme der Implementierung von Prinzipien nachhaltiger Entwicklung offen bleibt und die Anpassung an die konkreten Bedingungen jeder Organisation erfordert; die Verwirklichung der Nachhaltigkeits-Prinzipien kann von passiven Handlungen, die nur die Antwort auf äußere Herausforderungen und Erwartungen sein können, bis zu ganzheitlichen Programme, die sowohl innere als auch äußere Aspekte des Funtionierens der Unternehmen umfassen, reichen.

2. „Ausarbeitung einer Strategie, die als Grundlage nachhaltige Entwicklung hat ${ }^{\text {‘13 }}$ - die Verwirklichung der ökonomischen Ziele ist mit dem Erreichen anderer Ziele (gesellschaftliche, ökologische usw.) zu verbinden, was auf doppelte Weise verwirklicht werden kann: (a) durch das Berücksichtigen und Voraussetzen bestimmter Folgen wirtschaftlicher Handlungen und die Beseitigung bzw. Minimierung negativer Folgen; (b) durch die Vorbereitung einer eigenen Strategien, die die Prinzipien und Kriterien der Nachhaltigkeit umfasst.

3. „Nachhaltige Entwicklung ist in alle Aspekte der konkreten organisatorischen Handlung einzubauen"14 - das ist ein Resultat der oben genannten ganzheitlichen Erfordernisse; es erlaubt bestimmte Analysen und Reflexion, weil die Verwirklichung

\footnotetext{
${ }^{10}$ Ibidem

${ }^{11}$ Vgl. R. Janikowski: Wymiary zrównoważonego rozwoju. Rozwój lokalny, gospodarka przestrzenna, zdrowie środowiskowe, innowacyjność (Dimensionen nachhaltiger Entwicklung. Lokale Entwicklung, Umweltwirtschaft, Umweltgesundheit, Innovationsfähigkeit). WrocławPoznań 2010, S. 162ff.

${ }^{12}$ Ibidem, S. 162.

${ }^{13}$ Ibidem, S. 162.

${ }^{14}$ Ibidem, S. 163.
} 
nachhaltiger Strategien nicht automatisch realisiert wird, um so mehr, als sie in konkreten Situationen mit vielen Ziel- und Wertkonflikten zusammenhängt.

4. „Handlungen sind wichtiger als Worte“"15 - die Schwierigkeiten mit der Verwirklichung der Normen und Kriterien von Nachhaltigkeit kann hin und wieder dazu führen, dass wirkliche Handlungen dadurch vorgetäuscht werden, dass man sie durch Sprechen über Nachhaltigkeit ersetzt; die Verwirklichung von Nachhaltigkeitsstrategien erfordert Ausdauer, weil diese Aufgabe nie ein Ende hat und man sie ständig realisieren muss.

5. „Berufung eines Organs, das für die Verwirklichung von Nachhaltigkeit in der Organisation Verantwortung tragen kann" ${ }^{\prime 16}$ - die Stufe der Kompliziertheit, die mit der Strategie der Nachhaltigkeit verbunden ist, erfordert besonders im Fall großer Firmen die Berufung spezieller Gruppen, die mit der Verwirklichung dieser Strategie befasst ist; die Annahme solcher Strategien erfordert auch sehr oft die Notwendigkeit der Einbeziehung bestimmten Gruppen von Stakeholdern für die Konsultation.

$6 . \quad$ „Festlegung klarer Prinzipien"17 - Handlungen im Bereich der Vorbereitung nachhaltiger Strategien erfordert Transparenz und gleichzeitig auch das Verständnis und die Akzeptanz durch alle Subjekte in der Firma.

7. „Einbeziehung aller Stakeholder“18 - diese Einbeziehung kann manchmal bestimmte Probleme verursachen, z.B. die Verlängerung von Entscheidungsprozessen, aber diese Prozesse sollten im Grunde genommen und in bezug auf Nachhaltigkeit ständig erfolgen, was die Herstellung ständiger Kommunikationskanäle zwischen der Organisation und den interessierten Personen erfordert.

8. „Ausnutzung des Humanpotenzials“19 - die Menschen bilden immer den wesentlichen Bestandteil jeder Firma, und die Gestaltung nachhaltiger Prinzipien und Strategien kann in dieser Hinsicht die Attraktivität von Firmen erhöhen und damit auch positive Bedeutung für die Gestaltung guter Beziehungen zwischen den Mitarbeiter und der Firma haben.

9. „Füge Dich in Netze ein“ ${ }^{20}$ - jede Firma handelt nicht in allen Bereichen, aber es existieren viele Institutionen, die bestimmte Handlungen im Bereich der nachhaltigen Entwicklung unterstützen können; wichtig ist deshalb deren Nutzung sowie die Stellung in unterschiedlichen Ranglisten.

10. „Anpassung der Wirtschaftssysteme an die in der Organisation existierenden Nachhaltigkeitsstrategien“21 - in jeder Firma existieren unterschiedliche Bereiche ihrer Tätigkeit, aber alle Verwaltungsweisen sind zu koordinieren; in dieser Hinsicht können die Kritereien und Normen der Nachhaltigkeit eine wichtige Rolle spielen.

Die oben genannten Prinzipien eines nachhaltigen Unternehmens kann man gleichzeitig auch auf die Konzeption der gesellschaftlichen Verantwortung beziehen. Die Gestaltung von Nachhaltigkeit und die Verwirklichung der Prinzipien gesellschaftlicher Verfantwortung verbinden sich und unterstützen einander. Es scheint, dass Nachhaltigkeit

\footnotetext{
${ }^{15}$ Ibidem, S. 163.

${ }^{16}$ Ibidem, S. 163

${ }^{17}$ Ibidem, S.163.

${ }^{18}$ Ibidem, S. 164.

${ }^{19}$ Ibidem, S. 164.

${ }^{20}$ Ibidem, , S. 164.

${ }^{21}$ Ibidem, S. 164.
} 
einen allgemeineren und mehr ganzheitlichen Charakter im Verhältnis zur Konzeprion gesellschaftlicher Verantwortung hat. Die Verwirklichung der Ziele nachhaltiger Entwicklung ist gleichzeitig mit der Erfüllung der Erfordernisse gesellschaftlicher Verantwortung zu verbinden. Es muss jedoch nicht umgekehrt sein: Die Verwirklichung der gesellschaftlichen Verantwortung muss nicht gleichzeitig zur Verwirklichung nachhaltiger Entwicklung führen. Die hier erscheinenden Spannungen haben unterschiedliche Wurzeln, aber in der Praxis soll man stets in Richtung der Verbindung beider Konzeptionen streben. Strategien nachhaltiger Entwicklung sind stärker in die Zukunft und auf bestimmte Ziele gerichtet, auch wenn man gleichzeitig nicht alle diese Ziele verwirklichen muss bzw. kann, weil das Streben nach konkreten Zielen für den Fall, dass diese Ziele (noch) offen ist, wichtig ist. Im Fall der gesellschaftlichen Verantwortung ist die Orientierung auf die Zukunft auch wichtig, aber hier ist die Orientierung auf die laufende Aufgaben und deren Folgen wichtiger. Im Fall der Verantwortung haben wir es nämlich sehr oft mit dem traditionellen Verständnis der Verantwortung zu tun, die bis heute nicht völlig überwunden wurde.

Das Verständnis von Verantwortung als eine Verantwortung ex post, d.h. nach der Handlung und nach bestimmten Handlungsfolgen, hat noch weiterhin eine dominierende Bedeutung. Das betrifft auch die Konzeption der gesellschaftlichen Verantwortung und die Modelle ihrer Verwirklichung ${ }^{22}$. Im Fall der gesellschaftlichen Verantwortung haben auch die destruktiven (unverantwortbaren) Handlungen von Menschen eine größere Bedeutung. Strategien nachhaltiger Entwicklung sind noch nicht so empfänglich für bestimmte und durch die Individuen verursachte Missbräuche. R. Janikowski weist auf das Beispiel der Schiffsbesitzer der „Costa“ hin, die die Erfordernisse von Nachhaltigkeit und die Prinzipien gesellschaftlicher Verantwortung realisieren. Die Katastrophe der „Costa Concordia“ als Ergebnis unverantwortlicher Handlungen des Schiffskapitäns erschütterte das Vertrauen in diese Firma als eine gesellschaftlich verantworlich agierende Firma und weniger als in eine, die Normen der Nachhaltigkeit realisiert. Mitverwirklichung von Nachhaltigkeit und gesellschaftlicher Verantwortung bildet eine Herausforderung nicht nur in bezug auf die wirtschaftliche Organisationen, sonden auch auf die ganze politische, gesellschaftliche und kulturelle Umgebung. Erforderlich ist die Aktivität nicht nur von der Seite bestimmter Organisationen, sondern auch von der Seite der anderen Subjekte, von interessierten Personen unterschiedlicher Stufe, denn schließlich ist die Verwirklichung von Nachhaltigkeitsprinzipien das lebendige Interesse aller: „Teilnahme von Bürgern an Entscheidungsprozessen ist die beste Weise für das Erreichen der gesellschaftlichen und politischen Unterstützung für die Verwirklichung der Normen und Prinzipien von Nachhaltigkeit. [...] Mitregieren ist ein Prozess mit bestimmten Mechanismen und Institutionen, mit denen Bürger und gesellschaftliche Gruppen ihre Prioritäten und Interessen ausdrücken und widersprüchliche Interessen verhandeln. Das Mitregieren soll gewährleisten, dass die Gestaltung von politischen,

\footnotetext{
${ }^{22}$ Man weist hier auf die Modelle „vor dem Profit“ und „,nach dem Profit“" hin, und in diesem Fall scheint es so, dass die Verantwortung „ex ante“ (vor dem Handeln) die größere Bedeutung haben kann. Vgl. M. Rybak: Etyka menedżera - społeczna odpowiedzialność przedsiębiorstwa (Ethik von Managern - gesellschaftliche Verantwortung des Unternehmens). Warszawa 2004. Die Unterscheidung der Verantwortung ,ex ante“ und ,ex post“ (für das, was getan wurde) hängt mit der Konzeption von H. Jonas zusammen. Vgl. H. Jonas: Zasada odpowiedzialności (Das Prinzip Verantwortung). Kraków 1996.
} 
gesellschaftlichen und wirtschaftlichen Prioritäten im Grunde genommen einen gesellschaftlichen Konsens hat, in Übereinstimmung mit den Interessen von Frauen und Männer, von Reichen und Armen, von Vollberechtigten und nicht Vollberechtigten transparent und effektiv an den Entscheidungsprozessen vollzogen wurden “23. Die Verwirklichung von Nachaltigkeit und geselslchaftlicher Verantwortung erfordert schließlich die ständige Verbreiterung und Vertiefung der direkten Demokratie, die auf dem Fundament der Aktivitäten unterschiedlicher gesellschaftlicher Subjekte gebaut sein soll. Die Verwirklichung dieser beiden Konzeptionen hängt von den unterschiedlichen Formen der Aktivität der Bürgergesellschaft ab, aber gleichzeitig kann man durch die Partizipation der Gesellchaft an den bestimmten Entscheidungen und Handlungen die gesellschaftliche Ressourcen wecken und erweitern. Die Gestaltung von Nachaltigkeit und gesellschaftlicher Verantwortung bildet den Bereich, in dem wirtschaftliche Organisationen und gesellschaftliche Gruppen mit- und zusammenarbeiten können, was schliesslich zu bestimmten politischen, wirtschaftlichen, gesellschaftlichen und kulturellen Veränderungen führen kann. Die Verbindung von Nachhaltigkeit und gesellschaftlicher Verantwortung hat aus dieser Sicht eine wichtige, aber auch mehrfache Bedeutung.

\section{LITERATUR:}

[1] Borys T. (Hrsg.): Wskaźniki ekorozwoju (Indikatoren der Ökoentwicklung). Białystok 1999

[2] Borys T. (Hrsg.): Wskaźniki zrównoważonego rozwoju (Indikatoren nachhaltiger Entwicklung). Warszawa-Białystok 2005.

[3] Evan W. M., Freeman R. E.: Spółka i osoby żywotnie zainteresowane. Kapitalizm kantowski (Aktiengesellschaft und Stakeholder. Kantischer Kapitalismus), w: L. V. Ryan, J. Sojka (red.): Etyka biznesu. Z klasyki współczesnej myśli amerykańskiej. Poznań 1997, S.185-205.

[4] Fobelova D., Fobel P.: Etyka i kultura w organizacji (Ethik und Kultur in der Organisation). Tychy 2007.

[5] Gasparski W. (Hrsg.): Europejskie standardy etyki i społecznej odpowiedzialności biznesu (Europäische Standards der Ethik und der gesellschaftlichen Verantwortung). Warszawa 2003

[6] ISO 26000: www.pkn.pl/iso-26000; www.iso.org/iso/iso26000.

[7] Janikowski R.: Wymiary zrównoważonego rozwoju. Rozwój lokalny, gospodarka przestrzenna, zdrowie środowiskowe, innowacyjność (Dimensionen nachhaltiger Entwicklung. Lokale Entwicklung, Raumwirtschaft, Umweltgesundheit, Innovationsfähigkeit). Wrocław-Poznań 2010.

[8] Jonas H.: Zasada odpowiedzialności (Das Prinzip Verantwortung). Kraków 1996.

[9] Kronenberg J., Bergier T. (Hrsg.): Wyzwania zrównoważonego rozwoju w Polsce (Herausforderungen nachhaltiger Entwicklung in Polen). Kraków 2010.

\footnotetext{
${ }^{23}$ Vgl. R. Janikowski: Wymiary zrównoważonego rozwoju. Rozwój lokalny, gospodarka przestrzenna, zdrowie środowiskowe, innowacyjność (Dimensionen nachhaltiger Entwicklung. Lokale Entwicklung, Umweltwirtschaft, Umweltgesundheit, Innovationsfähigkeit), op. cit., S. $25 \mathrm{ff}$.
} 
[10]Ocieczek W., Gajdzik B.: Społeczna odpowiedzialność przedsiębiorstw produkcyjnych (Gesellschaftliche Verantwortung von Produktionsunternehmen). Gliwice 2010.

[11]Papuziński A.: Nachhaltigkeitsdeutungen in der polnischen wissenschaftlichen Fachliteratur, in: G. Banse, [11] Kiepas A. (Hrsg.): Nachhaltige Entwicklung. Von der wissenschaftlichen Forschung zur politischen Umsetzung. Berlin 2005,

[12]Piątek Z.: Ekofilozofia (Ökophilosophie). Kraków 2008.

[13]Ryan L. V., Sojka J. (Hrsg.): Etyka biznesu. Z klasyki współczesnej myśli amerykańskiej (Wirtschaftsethik.Vom klassischen modernen amerikanischen Denken). Poznań 1997.

[14]Rybak M.: Etyka menedżera - społeczna odpowiedzialność przedsiębiorstwa (Managerethik - gesellschaftliche Verantwortung des Unternehmens). Warszawa 2004.

\section{SPOŁECZNA ODPOWIEDZIALNOŚĆ JAKO FUNDAMENT ZRÓWNOWAŻONEJ PRZEDSIĘBIORCZOŚCI}

Konkurencyjność w działalności gospodarczej budowana jest obecnie w przypadku poszczególnych organizacji nie tylko w oparciu o realizację odpowiednich wartości ekonomicznych, jak i również nie tylko poprzez osiąganie określonych wartości „wewnętrznych” ( np. jakość towarów, usług itp.). Jednym z wymogów „zewnętrznych” wobec różnych form przedsiębiorczości jest dzisiaj konieczność zrównoważonego rozwoju, a spełnianie jego wymagań narzuca konieczność powiązania celów i wartości ekonomicznych z ekologicznymi i społecznymi. Jedną z istotnych podstaw i narzędzi dla budowania zrównoważonej przedsiębiorczości może być społeczna odpowiedzialność przedsiębiorstw wraz z towarzyszącymi wskaźnikami i regulatorami (np. ISO 26000). Artykuł omawia koncepcje społecznej odpowiedzialności jako podstawę dla zrównoważonej przedsiębiorczości, co stanowi nie tylko wyzwanie dla organizacji gospodarczych, lecz także dla ich otoczenia społecznego, politycznego i kulturowego. Identyfikuje podstawowe przeszkody we wdrażaniu zasad zrównoważonego rozwoju i zasad społecznej odpowiedzialności biznesu na poziomie organizacji gospodarczych oraz zwraca uwagę na strukturalne podobieństwa między nimi. Idea społecznej odpowiedzialności budzi bowiem wiele kontrowersji natury teoretycznej i praktycznej. W toczących się dyskusjach nie udało się teoretycznie sformułować odpowiedniego rozumienia odpowiedzialności odnoszącej się do podmiotów zbiorowych ( instytucjonalnych), z uwagi an dominującą w tym względzie tradycję odnoszenia odpowiedzialności jedynie do podmiotów indywidualnych. Skutkuje to $\mathrm{w}$ konsekwencji problemami jakie pojawiają się także w procesie urzeczywistniania idei społecznej odpowiedzialności w praktyce. Jej powiazanie z ideą zrównoważonego rozwoju też nie rozwiązuje wielu problemów, lecz nie neguje to w ogólności pozytywnych skutków związanych z realizacją społecznej odpowiedzialności i zrównoważonego rozwoju.

Słowa kluczowe: zrównoważony rozwój, ekorozwój, społeczna odpowiedzialność przedsiębiorstw, etyka biznesu.

\section{SOCIAL RESPONSIBILITY AS THE BASIS OF A SUSTAINABLE} ENTERPRISE

The competitiveness in the economic action today is built not solely on the basis of the implementation of appropriate economic values or by achieving certain "internal" values such as quality standards for goods or services. In many business organizations. For a successful management rather the fulfillment of certain "external" requirements is crucial. One of these "external" demands that are placed on different forms of entrepreneurial 
activity today is the need for sustainable development. The fulfillment of the requirements of sustainable development requires a high degree of connection of economic goals and values with the environmental and social concerns. This paper addresses the key CSR approaches both as a basis for sustainable business as well as the possible contribution of business to sustainable development at all. This is based on an understanding that the principle of socially responsible business is not only the economic organizations themselves, but also their social, political and cultural environment with new challenges. Developed from the idea of a social responsibility of business - similar to the earlier model of sustainable development - many visions that bring a number of controversies, both on the theoretical and at the practical level. In the previous discussion has not been able to achieve a theoretical consensus on an adequate understanding of responsibility with respect to collective and corporate (institutional) actors. The biggest reason is the widespread traditional view that only individual subjects are capable of responsibly. The consequences are serious problems that arise at the level of everyday practice in the implementation of the idea of corporate social responsibility.

Keywords: Sustainability, Eco-Development, Corporate Social Responsibility, Business Ethics

DOI:10.7862/rz.2014.hss.38

Przesłano do redakcji: marzec 2014

Przyjęto do druku: październik 2014 